

\title{
ASSOCIATION BETWEEN HEPATITIS B SURFACE ANTIBODY SEROPOSITIVITY AND CORONARY ARTERY DISEASE
}

\author{
ALIREZA AMIRZADEGAN, GHOLAMREZA DAVOODI, MOHAMMAD ALI BOROUMAND, \\ SIROUS DARABYAN, MARIA RAISSI DEHKORDI, HAMIDREZA GOODARZYNEJAD
}

\section{ABSTRACT}

BACKGROUND: Specific infectious agents have been found to be related to the pathogenesis of coronary atherosclerosis. AIMS: We assessed the possible association between angiographically proven coronary artery disease (CAD) and hepatitis $B$ surface antibody (HBS Ab) seropositivity in a population with relatively high prevalence of hepatitis B virus (HBV) infection. SEITING AND DESIGN: This was a cross-sectional study. MATERIALS AND METHODS: We analyzed data from 830 consecutive subjects undergoing coronary angiography, including angiographic results reported by two cardiologists for inter-observer reliability and assessment of HBS Ab status determined by enzyme-linked immunosorbent assay (ELISA). STATISTICAL ANALYSIS USED: Chisquare test or Fisher's exact test, independent two-sample t test and the Pearson's Correlation Coefficient test were used, as required. Statistics were performed using SPSS software version 13 (SPSS, Chicago, IL). RESULTS: Two hundred forty-nine (30\%) subjects had normal angiogram or minimal CAD, and 581 (70\%) had significant CAD in at least one major coronary artery. In patients with CAD and in patients without angiographic evidence of significant atherosclerosis, $28.7 \%$ and $28.9 \%$ respectively were positive for $\mathrm{HBV}(\mathrm{P}=0.954)$. Mean $\mathrm{C}$-reactive protein levels in subjects with positive and negative $\mathrm{HBS}$ Ab were $10.77 \pm 8.37 \mathrm{mg} / \mathrm{L}$ versus $10.33 \pm 7.64 \mathrm{mg} / \mathrm{L}$ respectively $(P=0.465)$. However, $C$-reactive protein levels in CAD group were significantly higher $(P<0.001)$. CONCLUSIONS: O ur results suggested hepatitis B surface antibody seropositivity has no relationship with coronary artery disease. Moreover, no significant linear correlation exists between HBS Ab and C-reactive protein levels. However, as previously shown, C-reactive protein level in patients with coronary artery disease is significantly higher than in patients with normal coronary arteries.

Key words: C-reactive protein, coronary artery disease, hepatitis B surface antibody, infection, inflammation

Tehran Heart Center, Tehran Heart Center, Medical Sciences/ University of Tehran, Tehran, Iran

Correspondence:

Dr. Hamidreza Goodarzynejad, Tehran Heart Center, Medical Sciences/ University of Tehran, Tehran, Iran.

E-mail: Hami_nag@yahoo.com

\section{INTRODUCTION}

The causes of atherosclerosis are still a puzzle. Traditional and established risk factors of atherosclerosis, such as hypertension, diabetes, hyperlipidemia and cigarette smoking, 
offer significant but partial explanations of the severity of atherosclerosis; indicating a possibility of other, as yet unidentified, etiologic factors. ${ }^{[1]}$ Discovery of such factors and, most important, of their possible mechanism of action would have crucial implications for the development of therapeutic approaches leading to reduce the devastating impact of this pathology. Substantial evidence now exists indicating that inflammation plays an important role in atherosclerosis. ${ }^{[2-6]}$

In pathology studies, atherogenic acute ischemic syndrome is characterized by local inflammation that includes an inflammatory component. Activated inflammatory cells and mediators have prothrombic and matrixdegrading effects which can influence the development of atherosclerosis.

Given the central role of Inflammation in the genesis of vulnerable plaque, there has been increasing interest in an infectious etiology. Currently, it is not obvious whether the general inflammatory response to an infective pathogen is important for development of atherosclerosis or whether there are specific atherogenic microorganisms. ${ }^{[5]}$ Such microorganisms which may directly cause or accelerate atherosclerosis include chlamydia pneumoniae, cytomegalovirus, herpes simplex virus and helicobacter pylori. ${ }^{[7-14]}$ Periodontal pathogens have also been found in human atheromas. ${ }^{[15]}$ In contrast, several epidemiological studies have failed to demonstrate any link between infection and atherosclerotic disease. ${ }^{[16]}$

Among the hepatitis viruses, the hepatitis $A$, $B$ and $C$ have been investigated for possible atherosclerosis effects. However, data available on this matter are conflicting. The results of two studies suggest hepatitis $B$ and $C$ virus infection may be independent risk factors for carotid atherosclerosis; ${ }^{[17,18]}$ these results have not been confirmed by two other studies, ${ }^{[19,20]}$ and one study even produced contradictory results with hepatitis virus infections being protective against atherosclerosis. ${ }^{[21]}$

The aim of the present analysis was to evaluate associations between hepatitis B infection and the risk of atherosclerosis in an HBVpredisposed study population.

\section{MATERIALS AND METHODS}

\section{Study subjects and definitions of CAD risk} factors

This cross-sectional study consisted of individuals who were referred for coronary angiography due to chest pain, noninvasive tests compatible with myocardial ischemia or history of myocardial infarction. From May to August 2005, a total of 830 individuals were selected out of 993 consecutive patients undergoing selective coronary angiography (SCA) at our center. Patients with previous history of anti-hepatitis $B$ virus vaccination were excluded because of its effect on HBS Ab levels. Moreover, patients with fever, major trauma or infections and myocardial infarction within the preceding 14 days were excluded from the study because of the simultaneous measurement of $\mathrm{C}$-reactive protein (CRP) and the probable influence of these conditions on CRP levels. Informed written consent was obtained from all participants who approved the collection of blood samples for scientific research. Patients who had angiographic evidence of atherosclerosis ( $\geq 50 \%$ stenosis of $\geq 1$ major coronary artery) in their epicardial coronary tree were classified as having coronary artery disease (CAD). Blood samples were taken for various measurements in all patients.

Risk factors for CAD that were analyzed included age, being male, cigarette smoking, diabetes, hyperlipidemia, hypertension and elevated CRP level. A history of past and current cigarette smoking was obtained for each patient. Patients who currently smoked any kind of tobacco or who had quit smoking for less than 6 months were considered smokers. Patients were considered to have diabetes provided that they were taking insulin, or oral hypoglycemic agents, had previously received such treatments or were currently under diet to control the condition. Patients with lack of awareness of their past history of diabetes were defined according to the new WHO criteria. ${ }^{[22]}$ Hyperlipidemia was defined as plasma total cholesterol level $\geq 200 \mathrm{mg} / \mathrm{dl}$ and/ or LDL-cholesterol level $\geq 130 \mathrm{mg} / \mathrm{dl}$ or being on lipid-lowering drugs at the time of study. A repeated blood pressure greater than 140 $\mathrm{mmHg}$ systolic and $90 \mathrm{mmHg}$ diastolic on two or more occasions or the use of antihypertensive drugs at the time of investigation was defined as hypertension.

\section{Serological markers and other laboratory testing}

Serum samples obtained from all study subjects were frozen at $-80^{\circ} \mathrm{C}$, and aliquots were thawed when needed for specific tests. Serological marker of hepatitis B (anti-HBS) was determined by enzyme-linked immunosorbent assay (ELISA); commercially available ELISA kits were used. Anti-HBS IgG values of $\geq 10$ units $/ \mathrm{ml}$ were considered positive. Plasma CRP was measured by latex immunoturbidometric methodology on an automated clinical analyzer system, Synchrony CX4 (Beckman Diagnostics, Fullerton, CA).

Lipoprotein (LP) (a) was measured using enzyme- linked immunosorbent assay. Tota serum cholesterol and triglyceride were assessed using enzymatic method. HD cholesterol was also checked using direct method, while Friedwald formula was applied for measuring LDL cholesterol.

\section{Assessment of coronary artery disease}

Coronary angiography was performed from the percutaneous femoral approach using standard angiographic techniques. Multiple views wer obtained in all patients, with the left anterio descending (LAD), left circumflex (LCX) and right coronary $(R C A)$ arteries visualized in multiple views by using cine-angiographic equipment (Philips Integris H 3000, Holland). The coronary angiograms were recorded on compact discs (CDs). Angiogram scoring was performed by two independent cardiologists according to clinical vessel score and Gensin score. Inter- and intra-observer reliability was measured as more than $95 \%$.

\section{Clinical vessel score}

The angiograms were classified as revealing either no coronary lesion with more than $50 \%$ luminal stenosis; or as having one, two or three major epicardial coronary arteries with more than $50 \%$ luminal obstructions. Lef main stem (LMS) stenosis was regarded as one vessel.

If the LMS and the LAD and/ or LCX were affected, this was counted as 2 points. Degree 
of stenosis was defined as the greatest percentage reduction of luminal diameter in any view compared with the nearest normal segment and was determined visually.

\section{Gensini score}

This severity score has been described previously. ${ }^{[23]}$ Briefly, the coronary arterial tree was divided into segments with multiplying factors according to geographic functional importance of any given segment ( 5 for the left main stem to 0.5 for the most distal segments), as well as the percent reduction in lumen diameter. The roentgenographic appearance of concentric lesions and eccentric plaques was assigned a score $(0,1,2,4,8,16$ or 32) according to the degree of luminal stenosis. The sum of the segmental scores gives the Gensini score, which puts emphasis on the severity of the disease.

\section{Statistical analysis}

Results for normally distributed continuous variables are expressed as mean $\pm \mathrm{SD}$. For categorical data, Chi-square test or Fisher's exact test, if necessary, was used. All tests were two-sided. The t-test for continuous data was applied. Correlation coefficients between variables were determined using conventional Pearson's correlation analysis. Partially
Pearson's correlation value was also used to adjust for potential confounders. A $P$ value $<0.05$ was considered statistically significant. All statistical analyses were performed with SPSS software version 13 (SPSS Inc., IL, USA).

\section{RESULTS}

Of the 830 study subjects with mean age of $57.03 \pm 10.59$ years (ranging from 25 to 90 years), 529 (63.7\%) were men and 581 (70.0\%) had coronary atherosclerosis. Body mass index (BMI) was significantly higher in patients with CAD as compared to subjects with normal epicardial coronary arteries or minimal coronary lesions $(P<0.001)$. Traditional CAD risk factors, including age, being male, diabetes, hypertension, smoking and hyperlipidemia, were proved to be associated with the risk of CAD [Table 1]. Furthermore, elevated CRP levels were detected to be significantly higher in those with CAD compared to those without CAD $(11.02 \pm 8.46 \mathrm{mg} / \mathrm{L}$ vs. $9.06 \pm 5.81 \mathrm{mg} / \mathrm{L}$; $P<0.001)$. Anti-HBS seropositivity prevalence was $28.8 \%$ in CAD patients and $28.9 \%$ in nonCAD subjects ( $P=0.954$; Table 2$)$.

HBV seropositivity and risk factors of CAD Subjects with anti-HBS IgG values of $>10$ units $/ \mathrm{ml}$ were considered HBV seropositive. In

Table 1: Traditional cardiac risk factors in patients with or without coronary artery disease

\begin{tabular}{lccc}
\hline Characteristics & Significant $C A D^{*}(n=581)$ & Non-significant CAD $(n=249)$ & $P$-value \\
\hline Age (year) & $58.42 \pm 10.34$ & $53.80 \pm 10.49$ & $<0.001$ \\
Male sex & $409(70.4)$ & $120(48.2)$ & $<0.001$ \\
Body mass index $\left(\mathrm{Kg} / \mathrm{m}^{2}\right)$ & $28.39 \pm 5.59$ & $26.76 \pm 4.01$ & $<0.001$ \\
Smoking & $182(31.3)$ & $45(18.1)$ & 0.001 \\
Diabetes & $161(27.7)$ & $45(18.1)$ & 0.003 \\
Hypertension & $245(42.2)$ & $83(33.3)$ & 0.019 \\
Hyperlipidemia & $270(46.5)$ & $75(30.2)$ & $<0.001$ \\
Lipoprotein $(\mathrm{a})(\mathrm{mg} / \mathrm{dl})$ & $19.98 \pm 13.88$ & $17.12 \pm 11.43$ & 0.002 \\
CRP & $(\mathrm{mg} / \mathrm{l})$ & $9.06 \pm 5.81$ & $<0.001$ \\
\hline
\end{tabular}

Table 2: Significant coronary artery disease and sero-status of hepatitis B virus marker

\begin{tabular}{|c|c|c|c|c|}
\hline & $\begin{array}{c}\text { Total study population } \\
\qquad(n=830)\end{array}$ & $\begin{array}{c}\text { CAD patients } \\
(n=581)\end{array}$ & $\begin{array}{c}\text { Non-CAD patients } \\
(n=249)\end{array}$ & $P$-value \\
\hline 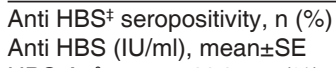 & $\begin{array}{l}239(28.8) \\
19.21 \pm 1.25\end{array}$ & $\begin{array}{c}167(28.7) \\
19.37 \pm 1.50\end{array}$ & $\begin{array}{c}72(28.9) \\
18.82 \pm 2.28\end{array}$ & $\begin{array}{l}0.954 \\
0.838\end{array}$ \\
\hline HBS Ag seropositivity, n (\%) & $12(1.4)$ & $7(1.2)$ & $5(2.0)$ & 0.375 \\
\hline
\end{tabular}
surface antigen.

the entire study population, there were totally 239 (28.8\%) HBS-seropositive individuals; of these, $170(71.1 \%)$ were male. As can be seen in Table 3, although seropositivity was not generally relative with traditional risk factors, anti-HBS Ab was associated with hyperlipidemia $(P=0.003)$ and diabetes $(P$ $=0.012$ ). Moreover, mean BMI was higher in seropositive participants compared to seronegative individuals $(27.61 \pm 4.28$ vs. 26.67 \pm 3.88; $P=0.002)$.

\section{CRP and HBV seropositivity}

Mean CRP levels were not significantly higher in HBV-seropositive $(10.77 \pm 8.37$ $\mathrm{mg} / \mathrm{L}$ ) individuals than in $\mathrm{HBV}$-seronegative individuals $(10.33 \pm 7.64 \mathrm{mg} / \mathrm{L})(P=0.465)$

Table 3: Baseline characteristics and atherosclerotic risk factors in participants with or without seropositivity for hepatitis B surface antibody

\begin{tabular}{|c|c|c|c|}
\hline Characteristics & $\begin{array}{c}\text { Anti-HBS*'-seropositive } \\
\text { patients }(n=239)\end{array}$ & $\begin{array}{c}\text { Anti-HBS-seronegative } \\
\text { patients }(n=591)\end{array}$ & $P$-value \\
\hline Age (years) & $57.18 \pm 10.41$ & $56.93 \pm 10.69$ & 0.759 \\
\hline Male sex & $170(71.1)$ & $365(61.8)$ & 0.011 \\
\hline Body mass index $\left(\mathrm{Kg} / \mathrm{m}^{2}\right)$ & $27.61 \pm 4.28$ & $26.67 \pm 3.88$ & 0.002 \\
\hline Smoking & $71(29.7)$ & $155(26.2)$ & 0.305 \\
\hline Diabetes & $45(18.8)$ & $160(27.1)$ & 0.012 \\
\hline Hypertension & $85(35.6)$ & $242(40.9)$ & 0.157 \\
\hline Hyperlipidemia & $81(33.9)$ & $268(45.3)$ & 0.003 \\
\hline Significant CAD & $160(66.9)$ & 417 (70.6) & 0.294 \\
\hline Cholesterol (mg/dl) & $205.88 \pm 50.56$ & $206.47 \pm 47.73$ & 0.878 \\
\hline $\mathrm{LDL}^{\dagger}$ cholesterol $(\mathrm{mg} / \mathrm{dl})$ & $130.01 \pm 40.53$ & $130.91 \pm 41.14$ & 0.781 \\
\hline $\mathrm{HDL}^{\ddagger}$ cholesterol (mg/dl) & $38.99 \pm 9.85$ & $39.28 \pm 10.06$ & 0.708 \\
\hline Triglyceride (mg/dl) & $184.54 \pm 121.72$ & $189.08 \pm 106.56$ & 0.601 \\
\hline Lipoprotein (a) (mg/dl) & $18.32 \pm 12.25$ & $19.43 \pm 13.67$ & 0.276 \\
\hline CRP§ (mg/l) & $10.77 \pm 8.37$ & $10.33 \pm 7.64$ & 0.465 \\
\hline $\operatorname{LVEF}^{\star \star}(\%)$ & $51.57 \pm 11.55$ & $51.48 \pm 11.33$ & 0.918 \\
\hline Gensini score, mean \pm SE & $43.45 \pm 3.24$ & $42.47 \pm 1.94$ & 0.787 \\
\hline
\end{tabular}
antibody; ' LDL: Low-density lipoprotein; ${ }^{*} H D L$ : High-density lipoprotein; ${ }^{~} \mathrm{CRP}$ : C-reactive protein; ${ }^{\star *} \mathrm{LVVEF}$ : Left ventricular ejection

Not surprisingly, traditional risk factors such as being male, age and hypertension were shown to be associated with increased CRP level (all $P$ values $<0.05)$, as reported previously. ${ }^{[24]}$

\section{HBV seropositivity and CAD}

The HBS Ab seropositivity was not significantly significant association between HBS A Since in our study, patients with epicardia normal coronary arteries were not distinguished from patients with minimal lesions, the correlation of Gensini score and CRP with HBS $A b$ was evaluated with separate Pearson's 
correlation tests to minimize a potential cause of bias in the results of patients with coronary atherosclerosis. No significant correlation was found between level of HBS Ab and Gensini score before $(r=-0.019, P=0.588)$ and after $(r=-0.016, P=0.654)$ adjustment for traditional cardiac risk factors. The correlation between HBS Ab and CRP levels was also not significant before $(r=0.024, P=0.488)$ and after $(r=0.019, P=0.590)$ adjustment for traditional cardiac risk factors.

\section{DISCUSSION}

In our investigation, we found no evidence to support an association between HBV seropositivity and CAD prevalence. These results are in concordance with Tong's et al. study, which also found no significant relationship between chronic hepatitis $B$ infection and development of coronary atherosclerosis in angiographically proven CAD patients. ${ }^{[25]}$ However, data from Japanese studies, derived from regular health checkups of employees (which is legally mandatory in Japan), demonstrated an increased prevalence of carotid atherosclerosis in HBV and HCV carriers. ${ }^{[17,18]}$ Different conclusions may have been arrived at due to variations in study designs, frequency of individuals with chronic hepatitis infection and possibly regional differences. More importantly, as Volzke et al. mentioned, the chosen approach to recruit study participants may have caused selection bias, because chronic hepatitis may lead to disadvantage in the employment market. ${ }^{[19]}$ An Italian study even showed a conflicting result that chronic active hepatitis with $B$ and $C$ viruses may help prevent atherosclerosis of the carotid, femoral and abdominal aorta arteries. ${ }^{[21]}$ Since all patients of the Italian study were known cases of chronic hepatitis, this may have given rise to false-positive impact of chronic viral hepatitis on carotid atherosclerosis. Additionally, patients recruited for the Italian study exhibited elevated liver enzymes; therefore, decreased plasma homeostasis resulting from impaired liver function may have protective effects regarding atherosclerosis in these patients.

There are several limitations related to our study, which should be noted. First, our nonCAD control group consisted of individuals who, on clinical evaluation, had suspected CAD. These individuals may not be considered as representative of subjects without $C A D$ who lack clinical features triggering a plan to perform coronary angiography. Second, although this study is among the largest studies on HBV infection and CAD to date, the present study is relatively small in size. As a result, we may have missed a very weak association between HBV seropositivity and coronary atherosclerosis.

Third, the possible and not assessed coinfections associated with vascular lesions (e.g., EBV, CMV, HIV-1, H. pylori) could interfere with the results in documented CAD patients. However, the patients were randomly selected, and we expect that this issue has not profoundly altered the result of analyses. Fourth, cases were defined only by use of serological characteristics, not by a more sensitive assay, i.e., the HBV DNA detection test. ${ }^{[26]}$ Finally, duration of the exposure could not be estimated; as in most cases, the finding of HBS Ab was accidental. As a result, short duration of inflammatory processes, as well as possible low intensity of infection, may cause failure in detecting atherosclerotic endpoints in some of the cases.

In conclusion, our results indicate that there was no statistical association between HBV infection and coronary atherosclerosis in the study group. Our findings disagree with previously reported findings that hepatitis $B$ infection is likely an important contributor to CAD.

Future studies are required to be conducted with a larger sample size and in patients without the presence of traditional risk factors for $C A D$ to confirm the findings.

\section{ACKNOWLEDGMENTS}

We are grateful to Dr. Sheikhfatollahi M. for providing statistical analysis. Our thanks are also due to Miss Ghassaby L. for typing the manuscript.

\section{REFERENCES}

1. Futterman LG, Lemberg L. Fifty percent of patients with coronary artery disease do not have any of the conventional risk factors. Am J Crit Care 1998;7:240-4.

2. Hansson GK. Inflammation, atherosclerosis and coronary artery disease. N Engl $\mathrm{J}$ Med 2005;352:1685-95.

3. Laroia ST, Ganti AK, Potti A. Role of infections in atherogenesis. Indian Heart J 2003;55:27-34.

4. Danesh J, Whincup $P$, Walker M, Lennon L, Thomson A, Appleby P, et al. Low grade inflammation and coronary heart disease: Prospective study and updated meta-analyses. BMJ 2000;321:199-204.

5. Muhlestein JB. Chronic infection and coronary artery disease. Med Clin North Am 2000;84:12348.
6. Sinha N, Garg N. Role of infections in the pathogenesis of atherosclerotic coronary artery disease: Past, present and future. Indian Heart $J$ 2000;52:79-85.

7. Goyal P, Kalek SC, Chaudhry R, Chauhan S, Shah N. Association of common chronic infections with coronary artery disease in patients without any conventional risk factors. Indian J Med Res 2007;125:129-36.

8. Vijayvergiya R, Agarwal N, Bahl A, Grover A, Singh M, Sharma M, et al. Association of Chlamydia pneumoniae and Helicobacter pylori infection with angiographically demonstrated coronary artery disease. Int J Cardiol 2006;107:428-9.

9. Bloemenkamp DG, Mali WP, Visseren FL, van der Graaf Y. Meta-analysis of seroepidemiologic studies of the relation between Chlamydia pneumoniae and atherosclerosis: Does study design influence results? Am Heart 2003;145:409-17.

10. Danesh J. Coronary heart disease, Helicobacte pylori, dental disease, Chlamydia pneumoniae an cytomegalovirus: Meta-analyses of prospective studies. Am Heart J 1999;138:S434-7.

11. Momiyama $\mathrm{Y}$, Ohmori $\mathrm{R}$, Taniguchi $\mathrm{H}$, Nakamura $\mathrm{H}$, Ohsuzu F. Association of Mycoplasma pneumoniae infection with coronary artery disease and its interaction with chlamydial infection. Atherosclerosis 2004;176:139-44.

12. Perumal V, Mathai E, Jose J, Gupta S. Prevalence of Chlamydia pneumoniae IgG antibodies in patients with coronary artery disease: A report from an Indian population. Indian Heart 2003;55:667.

13. Horvath R, Cerny J, Benedik J, Hokl J Jr, Jelinkova I, Benedik J. The possible role of human cytomegalovirus (HCMV) in the origin of atherosclerosis. J Clin Virol 2000;16:17-24.

14. Fong IW. Emerging relations between infectious diseases and coronary artery disease and atherosclerosis. CMAJ 2000;163:49-56.

15. Haraszthy VI, Zambon JJ, Trevisan M, Zied $M$, Genco RJ. Identification of periodontal 
pathogens in atheromatous plaques. J Periodontol 2000;71:1554-60.

16. Yetkin G. Is seropositivity enough to show the association between coronary artery disease and Chlamydia pneumoniae and Helicobacter pylori infection? Int J Cardiol 2006;113:432-4.

17. Ishizaka N, Ishizaka Y, Takahashi E, Tooda E, Hashimoto H, Nagai R, et al. Association between hepatitis $\mathrm{C}$ virus seropositivity, carotid-artery plaque and intima-media thickening. Lancet 2002;359:133-5.

18. Ishizaka N, Ishizaka Y, Takahashi E, Toda Ei E, Hashimoto H, Ohno M, et al. Increased prevalence of carotid atherosclerosis in hepatitis $B$ virus carriers. Circulation 2002;105:1028-30.

19. Völzke H, Schwahn C, Wolff B, Mentel R, Robinson DM, Kleine V, et al. Hepatitis B and C virus infection and the risk of atherosclerosis in a general population. Atherosclerosis 2004;174: 99-103.

20. Kiechl S, Egger G, Mayr M, Wiedermann CJ, Bonora E, Oberhollenzer F, et al. Chronic infections and the risk of carotid atherosclerosis: Prospective results from a large population study. Circulation 2001;103:1064-70.

21. Bilora F, Rinaldi R, Boccioletti V, Petrobelli F, Girolami A. Chronic viral hepatitis: A prospective factor against atherosclerosis: A study with echocolor Doppler of the carotid and femoral arteries and the abdominal aorta. Gastroenterol Clin Biol 2002;26:1001-4.

22. WHO Consultation Definition, diagnosis and classification of diabetes mellitus and its complications, Part 1: Diagnosis and classification of diabetes mellitus. World Health Organisation: Geneva; 1999.

23. Gensini GG. A more meaningful scoring system for determining the severity of coronary heart disease. Am J Cardiol 1983;51:606.

24. Rohde LE, Hennekens $\mathrm{CH}$, Ridker PM. Survey of $\mathrm{C}$-reactive protein and cardiovascular risk factors in apparently healthy men. Am J Cardiol 1999;84:1018-22.

25. Tong DY, Wang XH, Xu CF, Yang YZ, Xiong $S D$. Hepatitis $B$ virus infection and coronary atherosclerosis: Results from a population with relatively high prevalence of hepatitis $B$ virus. World J Gastroenterol 2005;11:1292-6.

26. Bréchot C, Thiers V, Kremsdorf D, Nalpas B, Pol S, Paterlini-Bréchot P. Persistent hepatitis B infection in subjects without hepatitis $B$ surface antigen: Clinically significant or purely occult? Hepatology 2001;34:194-203.

Source of Support: Nil, Conflict of Interest: None declared. 